\title{
Charge ordering and dielectric properties in the near half-doped $\mathrm{Pr}_{0.79} \mathrm{Na}_{0.21} \mathrm{MnO}_{3}$ perovskite
}

\author{
M. Sánchez-Andújar, S. Yáñez-Vilar, S. Castro-García, M.A. Señarís- \\ Rodríguez'
}

Departamento Química Fundamental, Facultad de Ciencias, Universidade da Coruña, 15071 A Coruña, Spain

Journal of Solid State Chemistry

Volume 181, Issue 6, June 2008, Pages 1354-1360

Received 14 December 2007, Revised 11 February 2008, Accepted 10 March 2008, Available online 16 March 2008

DOI: 10.1016/j.jssc.2008.03.004

\begin{abstract}
The $\mathrm{Pr}_{0.79} \mathrm{Na}_{0.21} \mathrm{MnO}_{3}$ perovskite has been prepared in polycrystalline form by a ceramic method. We have carried out its structural characterization by synchrotron X-ray powder diffraction (SXRPD) at room temperature and at $150 \mathrm{~K}\left(T<T_{\mathrm{CO}}\right)$. In the charge ordering (CO) state, the SXRPD data can be refined both on the basis of the sitecentred model and the bond-centred model proposed for half-doped manganites, without a clear advantage of one model over the other. From the dielectric point of view, at the $\mathrm{CO}$ temperature this manganite shows a maximum in the dielectric constant whose origin is intrinsic, and cannot be attributed to the presence of extrinsic factors. We relate this dielectric behaviour to the formation of polar entities at the temperature of charge condensation, due to an asymmetric charge distribution intermediate between site-centred and bond-centred type.
\end{abstract}




\section{Graphical abstract}

We have prepared the near half-doped $\operatorname{Pr}_{0.79} \mathrm{Na}_{0.21} \mathrm{MnO}_{3}$ perovskite, whose SXRPD data can be refined both on the basis of the site-centred and bondcentred model in the CO state. Its dielectric constant shows an anomaly at $T_{\text {co }}$ whose origin is intrinsic and that we relate to the formation of polar entities at the temperature of charge condensation due to an asymmetric intermediate charge distribution.

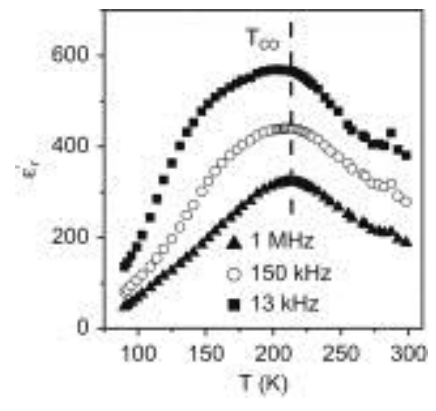

\section{Keywords}

Perovskite; Half-doped manganite; Charge-ordering; Dielectric behaviour

\section{Introduction}

The electronic phenomenon of charge ordering in transition metal oxides has gained renewed attention in the last years in connection with the interesting new properties and phenomenologies displayed by compounds belonging to this group, such as colossal magnetoresistance (CMR) in $\mathrm{CO}$ manganese perovskites [1], phase separation [2], etc. More recently, a very interesting debate has arisen about the most adequate description for their charge-ordered ground-state structure.

In that context, the traditional picture for charge ordering in transition metal oxides, that relies on the idea of localization and ordering of extra electrons or holes on a specific metal sublattice, is being questioned[3] and [4].

Let us take the case of the intensively studied $\mathrm{CO}$ half-doped manganites $L n_{1-x} M_{x} \mathrm{MnO}_{3}\left(L n^{3+}\right.$ : La, rare earth with $x=0.5$ when $M^{2+}$, and $x=0.25$ when $\left.M^{+}\right)$.

According to the traditional picture, initially proposed in the pioneering work of Goodenough [5], in the charge ordered state, $\mathrm{Mn}^{3+}$ and $\mathrm{Mn}^{4+}$ ions alternate in the lattice giving rise to a checkerboard pattern. In addition, orbital ordering of the $d_{z}{ }^{2}$ orbital of the $\mathrm{Mn}^{3+}$ cations gives rise to the associated CE-type magnetic structure [5]. 
Crystallographically, in the CO state these half-doped manganites show lower symmetry than in the delocalized state $\left(T>T_{\mathrm{CO}}\right)$, where the structure is usually orthorhombic (S.G.: Pnma) with cell parameters $\approx \sqrt{ } 2 a_{p} x \approx 2 a_{p} x \approx \sqrt{ } 2 a_{p}\left(a_{p} \approx 3.8 \AA\right.$ ).

In that context, the most widely used structural model for $T<T_{\mathrm{CO}}$ is that of Radaelli et al. [6], that supports the Mn-centred charge ordering scenario, and that was initially proposed to refine the powder X-ray and neutron powder diffraction data of $\mathrm{La}_{0.50} \mathrm{Ca}_{0.50} \mathrm{MnO}_{3}$ and subsequently extended to other $\mathrm{CO}$ half-doped manganites [7].

This Radaelli's model proposes for $T<T_{\text {CO }}$ a monoclinic $P 2_{1} / m$ symmetry $\approx 2 \sqrt{ } 2 a_{p} x \approx 2 a_{p} x \approx \sqrt{ } 2 a_{p}$ superstructure, with two displacement coordinates to describe the charge and orbital ordering modulation superimposed on the parent Pnma structure. It should be indicated that the application of this model reveals that the actual degree of charge localization in half-doped manganites is always much reduced from the ideal values, below $50 \%$ according to recent results [8].

In 2002, an alternative structural model was proposed by Daoud-Aladine et al. on the basis of a neutron diffraction study of a single crystal of $\operatorname{Pr}_{0.6} \mathrm{Ca}_{0.4} \mathrm{MnO}_{3}[9]$. According to these authors in the $\mathrm{CO}$ state the crystal shows an orthorhombic $P 2{ }_{1} n m$ pseudosymmetry (the real structure is in fact monoclinic $P 11 \mathrm{~m}$ ) with cell parameters $\approx 2 \sqrt{ } 2 a_{p} x \approx 2 a_{p} x \approx \sqrt{ } 2 a_{p}$ and two crystallographically non-equivalent $\mathrm{Mn}$ sites. Very interestingly $\mathrm{Mn}$ ions in both sites show the same charge and an average valence of $3.5+$. These results are consistent with a bond-centred superstructure based on an alternative Zener polaron ordering model (ZPO), in which the extra electron is localized not on metal sites but on bonds, in this case on pairs of Mn ions [4] and [9].

Moreover, other authors on the basis of theoretical studies have recently suggested that an alternative type of ordering is possible in $R_{1-x} \mathrm{Ca}_{x} \mathrm{MnO}_{3}$ around $x=0.50$ and other transition metal compounds: a type of charge ordering that is intermediate between site-centred and bond-centred [10].

Most interestingly, these authors have also predicted that such intermediate situation can lead to a non-centrosymmetric charge distribution and to a net polarization [10], giving thus rise to a new electronic ferroelectric (FE) state.

In that context, previous experimental studies on a $\operatorname{Pr}_{0.67} \mathrm{Ca}_{0.33} \mathrm{MnO}_{3}$ polycrystalline sample [11] and single crystal [12], and in a $\mathrm{La}_{1.5} \mathrm{Sr}_{0.5} \mathrm{NiO}_{4}$ ceramic [13] have already revealed anomalies in the dielectric constant of these compounds at the $\mathrm{CO}$ 
temperature, feature that could be related to such electronic transition and thus support the picture of a new FE state.

Other good candidates to show such behaviour could be found around the half-doped manganite $\operatorname{Pr}_{0.75} \mathrm{Na}_{0.25} \mathrm{MnO}_{3}$, a $\mathrm{CO}$ compound with $T_{\mathrm{CO}} \sim 220 \mathrm{~K}$, that is a Mott insulator close to an edge between a localized-delocalized state.

This manganite belongs to the series of rare earth manganese perovskites doped with univalent alkaline cations, that have been much less studied than the corresponding compounds doped with alkali-earth cations [14], [15] and [16].

For example, structural data of the CO state have only been reported in the case of the $x=0.20$ sample at $T=7 \mathrm{~K}$; and they have just been refined on the basis of the room temperature Pnma structure and neglecting eventual superstructures in the $\mathrm{CO}$ phase [14], that remain to be characterized.

In the present study we deepen in the structural characteristics of the CO state in the near half-doped $\operatorname{Pr}_{0.79} \mathrm{Na}_{0.21} \mathrm{MnO}_{3}$ perovskite. Also, we study the dielectric behaviour of this compound looking for special features that could be related to the electronic charge ordering transition.

\section{Experimental}

The ceramic method was used to prepare a sample with nominal composition $\operatorname{Pr}_{0.75} \mathrm{Na}_{0.25} \mathrm{MnO}_{3}$. For this purpose, stoichiometric amounts of $\operatorname{Pr}_{6} \mathrm{O}_{11}$ (Aldrich, 99.9\%), $\mathrm{NaHCO}_{3}$ (Panreac, 99\%) and $\mathrm{MnCO}_{3}$ (Aldrich, 99.9\%) were mixed and heated at $900{ }^{\circ} \mathrm{C} / 24 \mathrm{~h}$, and then pressed into pellets and treated at $1100{ }^{\circ} \mathrm{C} / 48 \mathrm{~h}$ in air and cooled to room temperature at $0.7^{\circ} \mathrm{C} / \mathrm{min}$.

The obtained material was characterized by conventional X-ray powder diffractometry (XRPD) in a Siemens D-5000 diffractometer at room temperature and using CuKa $=1.5418$ Å radiation.

In addition, synchrotron X-ray powder diffraction (SXRPD) was carried out at room temperature and $150 \mathrm{~K}$ on the BM25A beamline $(\lambda=0.8261 \AA$ ) at the ESRF (Grenoble, France). The sample was loaded in a borosilicate capillary $(\varphi=0.3 \mathrm{~mm})$ and rotated during data collection. The Rietveld analyses were performed with the GSAS 
program [17], where the peak shapes were described by a pseudo-Voigt function (CW profile function 3) and the background was modelled with an 8-term polynomial.

The formal valence of manganese atoms was calculated from the measured bond distances by the Bond Valence Sum (BVS) method [18] using the softBV program [19].

Magnetic properties were studied in a Quantum Design MPMS Squid magnetometer. In this context, zero-field-cooled (ZFC) and field-cooled (FC) magnetic susceptibility data were obtained under a field of 100 Oe in the temperature range $5 \leqslant T(\mathrm{~K}) \leqslant 300$.

The four-probe electrical dc resistivity of pressed pellets was measured as a function of temperature in the range $130 \leqslant T(\mathrm{~K}) \leqslant 315$ in a homemade device.

The complex dielectric permittivity of the samples was measured with a parallel-plate capacitor coupled to a precision LCR-meter Agilent $4284 \mathrm{~A}$ capable to measure in the frequency range $20 \leqslant v(\mathrm{~Hz}) \leqslant 10^{6}$. The capacitor was mounted in an aluminium box refrigerated with liquid nitrogen, incorporating a mechanism to control the temperature up to $300 \mathrm{~K}$. The pelletized sample, with an area of $23 \mathrm{~mm}^{2}$ and thickness of $0.8 \mathrm{~mm}$, was prepared to fit into the capacitor and gold was sputtered on its surfaces to ensure good electrical contact with the electrodes of the capacitor. Additional measurements using sputtered silver contacts were also carried out.

To test the optimal performance of the experimental set-up, a commercial $\mathrm{SrTiO}_{3}$ sample was measured and values similar to those reported in the literature [20] were obtained.

Impedance complex plane plots were analysed using the LEVM program [21].

\section{Results and discussion}

\subsection{General characterization}

According to the conventional X-ray powder diffraction results this sample is single phase and contains the desired phase.

In order to check its quality, we studied its magnetic and transport properties to confirm that it presents the transitions reported in the literature for this compound [14], even if this procedure does not unambiguously allow to differentiate between the $x=0.20-0.25$ compounds that seem to show a very similar magnetic and electric behaviour [14]. 
In Fig. 1a we show the temperature dependence of the ZFC and FC susceptibility of this sample, results that are similar to those obtained by other authors [14]. As it can be seen, in the FC curve a small cusp appears at $\sim 220 \mathrm{~K}$ followed by a small shoulder at $\sim 170 \mathrm{~K}$, features that, according to Hejtmánek et al. [14], correspond to the CO transition and the AF order Néel temperature, respectively. At lower temperatures, below $T_{\mathrm{g}} \sim 40 \mathrm{~K}$ (see inset in Fig. 1a) the ZFC and FC curves split, due to the formation of a spin glass state due to a competition between AF and FM interactions [15].

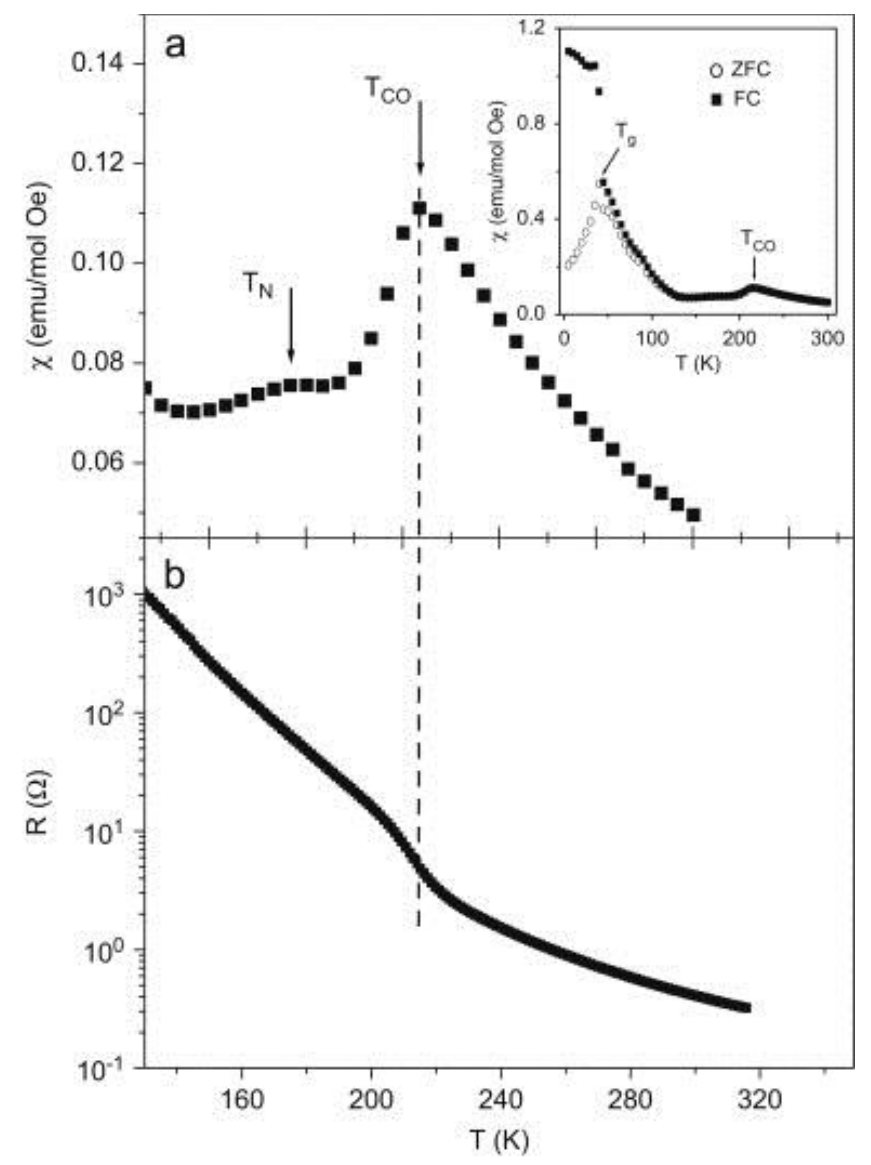

Fig. 1. (a) Field-cooled (FC) susceptibility versus temperature curve corresponding to the temperature interval $130<T(K)<315$ under a field of 100 Oe. Inset: Field-cooled (FC) and zero-field-cooled (ZFC) susceptibility data for the larger temperature interval $5<T(\mathrm{~K})<300 \quad(H=100 \mathrm{Oe})$. (b) The dc resistivity versus temperature $(135<T(K)<300)$.

The dc resistivity $(\rho)$ of this sample as a function of temperature is shown in Fig. $1 \mathrm{~b}$, where the expected jump in $\rho$ at the charge ordering transition is clearly observed at $T_{\mathrm{CO}} \approx 220 \mathrm{~K}$.

\subsection{Detailed structural characterization}

The SXRPD pattern obtained at room temperature was refined in the conventional orthorhombic symmetry (S.G.: Pnma), with cell parameters $\approx \sqrt{ } 2 a_{p} x \approx 2 a_{p} x \approx \sqrt{ } 2 a_{p}\left(a_{p} \approx 3.8 \AA\right)$. 
The degree of occupation of the $\mathrm{Pr}$ and $\mathrm{Na}$ sites was also included in the refinement. As a result, a small deficiency in the compound's sodium content was detected. This implies that a certain sodium loss has occurred during the synthesis, as also observed by other authors in their samples [16]. This provokes a small shift in the $\mathrm{Pr} / \mathrm{Na}$ ratio with respect to the nominal values so that the real stoichiometry of the compound turns to be $\operatorname{Pr}_{0.79} \mathrm{Na}_{0.21} \mathrm{MnO}_{3}$. This sodium deficiency also implies an excess of manganese in the sample, that we have finally found in the form of $\mathrm{Mn}_{3} \mathrm{O}_{4}$ (hausmanite) even if present in a very small amount $(<2 \%)$.

The obtained lattice parameters, refined atomic coordinates and derived interatomic distances are summarized in Table 1 and Table 2. As shown in Table 2, in this structure there is only one site for the Mn cations that are located in an octahedral environment with three different $\mathrm{Mn}-\mathrm{O}$ bond lengths.

Table 1. Structural parameters after the Rietveld refinement of the SXRPD pattern with the conventional orthorhombic symmetry (S.G.:Pnma) of the data obtained at room temperature The estimated errors are given in parentheses.

\begin{tabular}{|l|l|l|l|l|l|}
\hline \multirow{2}{*}{ Atom } & \multicolumn{2}{|l|}{$a=5.4312(1) \AA, b=7.6825(2) \AA, c=5.4414(1) \AA$} & \multirow{U}{*}{ O $\left.\AA^{2}\right)$} & \multirow{2}{*}{ Occ. } \\
\cline { 2 - 4 } & $x$ & $y$ & $z$ & \\
\hline $\mathrm{Pr}$ & $0.0242(1)$ & 0.25 & $-0.0037(3)$ & $0.0162(1)$ & $0.79(1)$ \\
\hline $\mathrm{Na}$ & $0.0242(1)$ & 0.25 & $-0.0037(3)$ & $0.0162(1)$ & $0.21(1)$ \\
\hline $\mathrm{Mn}$ & 0 & 0 & 0.5 & $0.0138(1)$ & 1 \\
\hline $\mathrm{O} 1$ & $0.4933(10)$ & 0.25 & $0.0624(22)$ & $0.016(2)$ & 1 \\
\hline O2 & $0.2843(15)$ & $0.0343(12)$ & $0.7195(16)$ & $0.029(1)$ & 1 \\
\hline & $R_{\mathrm{wp}}=4.27, R_{\mathrm{p}}=3.43, X^{2}=2.61$ & & \\
\hline
\end{tabular}

Table 2. Mn-O bond distances obtained from the room temperature refinement and valences determined from the Bond Valence Sum (BVS) method for Mn atoms The estimated errors are given in parentheses.

\begin{tabular}{|l|l|}
\hline & Bond length $(\AA)$ \\
\hline $\mathrm{Mn}-\mathrm{O}(1)$ & $1.950(2)$ \\
\hline $\mathrm{Mn}-\mathrm{O}(2 a)$ & $1.972(9)$ \\
\hline $\mathrm{Mn}-\mathrm{O}(2 b)$ & $1.941(10)$ \\
\hline $\mathrm{BVS}$ & 3.34 \\
\hline
\end{tabular}

These results are in agreement with the data reported in the literature [22]. 
On the other hand, the SXRPD pattern obtained at $150 \mathrm{~K}\left(T<T_{\mathrm{CO}}\right)$ cannot be fully indexed on the basis of the room temperature orthorhombic cell due to the existence of a few extra reflexions (see inset in Fig. 2).

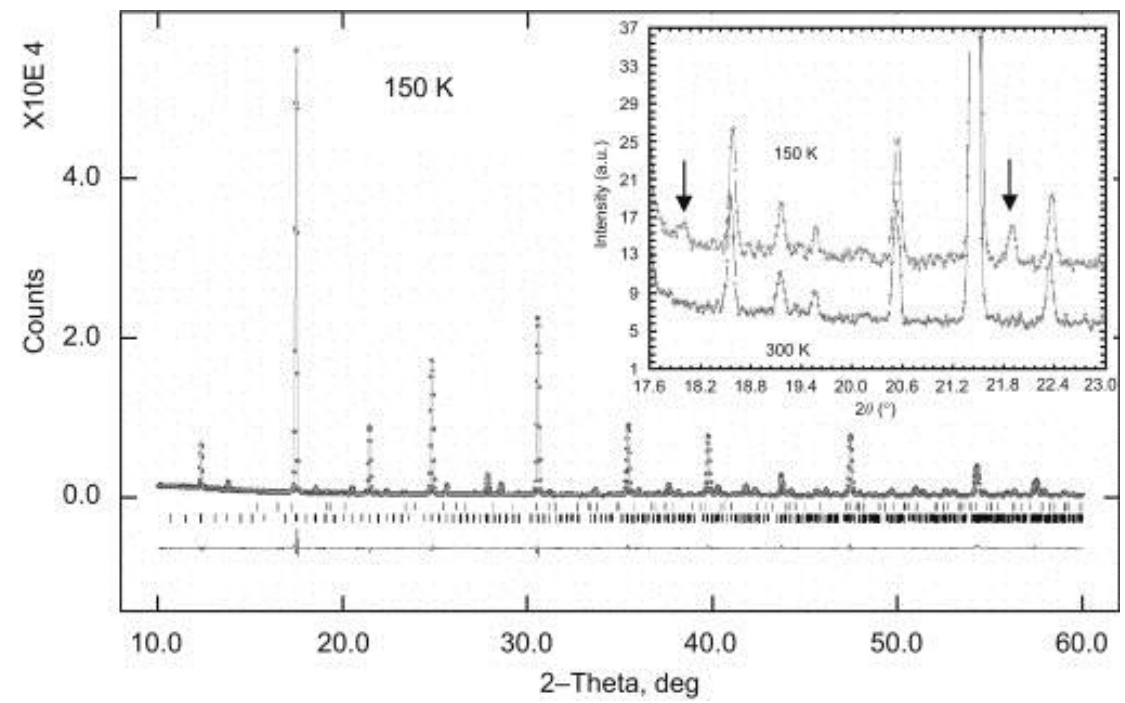

Fig. 2. Rietveld refinement of the SXRPD pattern of $\operatorname{Pr}_{0.79} \mathrm{Na}_{0.21} \mathrm{MnO}_{3}$ at $150 \mathrm{~K}\left(T<T_{\mathrm{CO}}\right)$ to the site-centred model. Key: Observed data (circles) and calculated profile (solid line); the difference plot is drawn below the profile. Lower tick marks indicate peak positions of the $\mathrm{Pr}_{0.79} \mathrm{Na}_{0.21} \mathrm{MnO}_{3}$ compound and upper tick marks of the $\mathrm{Mn}_{3} \mathrm{O}_{4}$ compound. Inset: Comparison of the SXRPD pattern at 300 and $150 \mathrm{~K}$, where the arrows indicate the extra peaks present in the low-temperature phase.

So we tried different structural models when carrying out its Rietveld refinement looking for the best fit, starting with the Radaelli's site-centred model [6] and continuing with the bond-centred model of Daoud-Aladine et al. [9], as we will show below.

We have to indicate that for these refinements the degree of $\mathrm{Pr}$ and $\mathrm{Na}$ occupation was fixed to the values obtained at room temperature and that the thermal parameters $U$ were constrained to be equal for similar atomic species.

In order to refine our pattern on the basis of the site-centred model we used as starting atomic coordinates those reported in the work of Goff et al. for the $\operatorname{Pr}_{0.5} \mathrm{Ca}_{0.5} \mathrm{MnO}_{3}$ perovskite [23].

The resulting SXRPD refinement at $150 \mathrm{~K}$ is shown in Fig. 2 and the obtained lattice parameters, refined atomic coordinates, derived interatomic distances and agreement factors are summarized in Table 3 and Table 4. 
Table 3. Structural parameters after the Rietveld refinement of the SXRPD pattern with the sitecentred model (S.G.: $P 2_{1} / \mathrm{m}$ ) of the data obtained at $T=150 \mathrm{~K}$ The estimated errors are given in parentheses.

\begin{tabular}{|c|c|c|c|c|}
\hline \multirow[t]{2}{*}{ Atom } & \multicolumn{3}{|c|}{$a=10.8513(2) \AA \AA$} & \multirow[t]{2}{*}{$U /\left(\AA^{2}\right)$} \\
\hline & $x$ & $y$ & $z$ & \\
\hline $\mathrm{Pr} / \mathrm{Na}(1)$ & $0.0122(7)$ & 0.2500 & $-0.0017(13)$ & $0.0039(1)$ \\
\hline $\mathrm{Pr} / \mathrm{Na}(2)$ & $0.2611(6)$ & 0.25000 & $0.5156(6)$ & $0.0039(1)$ \\
\hline $\mathrm{Pr} / \mathrm{Na}(3)$ & $0.5128(7)$ & 0.25000 & $-0.0033(12)$ & $0.0039(1)$ \\
\hline $\mathrm{Pr} / \mathrm{Na}(4)$ & $0.7636(7)$ & 0.25000 & $0.4961(6)$ & $0.0039(1)$ \\
\hline $\operatorname{Mn}(1)$ & $0.2488(12)$ & $-0.0020(12)$ & $0.0060(14)$ & $0.0030(2)$ \\
\hline $\mathrm{Mn}(2 a)$ & 0.0000 & 0.0000 & 0.5000 & $0.0030(2)$ \\
\hline $\operatorname{Mn}(2 b)$ & 0.5000 & 0.0000 & 0.5000 & $0.0030(2)$ \\
\hline 01 & $0.016(5)$ & 0.2500 & $0.436(9)$ & $0.0069(3)$ \\
\hline $\mathrm{O} 2$ & $0.228(4)$ & 0.2500 & $0.064(8)$ & $0.0069(3)$ \\
\hline $\mathrm{O} 3$ & $0.486(4)$ & 0.2500 & $0.451(10)$ & $0.0069(3)$ \\
\hline $\mathrm{O} 4$ & $0.755(5)$ & 0.2500 & $0.066(9)$ & $0.0069(3)$ \\
\hline O5 & $0.357(2)$ & $-0.060(4)$ & $0.300(4)$ & $0.0069(3)$ \\
\hline O6 & $-0.114(3)$ & $0.038(5)$ & $0.755(5)$ & $0.0069(3)$ \\
\hline O7 & $0.859(3)$ & $-0.017(4)$ & $0.254(6)$ & $0.0069(3)$ \\
\hline O8 & $0.402(4)$ & $0.023(4)$ & $0.826(5)$ & $0.0069(3)$ \\
\hline
\end{tabular}

Table 4. Selected interatomic distances $(\AA)$ in $\mathrm{Pr}_{0.79} \mathrm{Na}_{0.21} \mathrm{MnO}_{3}$ at $T=150 \mathrm{~K}$ obtained from the Rietveld refinement to the site-centred model and valences determined from the Bond Valence model for Mn atoms The errors are given in parentheses.

\begin{tabular}{|l|l|}
\hline & Bond length (Å) \\
\hline $\mathrm{Mn} 1-\mathrm{O} 2$ & $1.914(17)$ \\
\hline $\mathrm{Mn} 1-\mathrm{O} 4$ & $2.002(18)$ \\
\hline $\mathrm{Mn} 1-\mathrm{O} 5$ & $2.045(29)$ \\
\hline $\mathrm{Mn} 1-\mathrm{O} 6$ & $1.992(34)$ \\
\hline $\mathrm{Mn} 1-\mathrm{O} 7$ & $1.847(10)$ \\
\hline $\mathrm{Mn} 1-\mathrm{O} 8$ & $1.920(40)$ \\
\hline $\mathrm{BVS}(\mathrm{Mn} 1)$ & 3.47 \\
\hline $\mathrm{Mn}(2 a)-\mathrm{O} 1\left(x_{2}\right)$ & $1.958(11)$ \\
\hline $\mathrm{Mn}(2 a)-\mathrm{O} 6\left(x_{2}\right)$ & $1.876(33)$ \\
\hline $\mathrm{Mn}(2 a)-\mathrm{O} 7\left(x_{2}\right)$ & $2.032(29)$ \\
\hline $\mathrm{BVS}(\mathrm{Mn} 2 a)$ & 3.27 \\
\hline $\mathrm{Mn}(2 b)-\mathrm{O} 3\left(x_{2}\right)$ & $1.943(9)$ \\
\hline $\mathrm{Mn}(2 b)-\mathrm{O} 5\left(x_{2}\right)$ & $1.950(29)$ \\
\hline $\mathrm{Mn}(2 b)-\mathrm{O} 8\left(x_{2}\right)$ & $2.080(29)$ \\
\hline $\mathrm{BVS}(\mathrm{Mn}(2 b))$ & 3.01 \\
\hline
\end{tabular}


In this monoclinic structure (S.G.: $P 2_{1} / m$ ), there are three non-equivalent crystallographic sites for the Mn cations: $\mathrm{Mn}(1)$ in (4f) Wyckoff sites, $\mathrm{Mn}(2 a)$ in $(2 c)$ sites and $\mathrm{Mn}(2 b)$ in $(2 d)$ sites (see Table 4).

Charges at these three sites have been estimated from the measured bond distances using the BVS method. The so-calculated manganese formal valences, that are also included in Table 4, reveal that the $\mathrm{Mn}(1)$ cations show the highest BVS (3.47) while the $\mathrm{Mn}(2 a)$ and $\mathrm{Mn}(2 b)$ cations show lower BVS values (3.27 and 3.01, respectively). These results indicate that in the $\mathrm{Mn}(1)$ site the cations are more $\mathrm{Mn}^{4+}$-like, while those of $\mathrm{Mn}(2 a)$ and $\mathrm{Mn}(2 b)$ sites are more $\mathrm{Mn}^{3+}$-like, even if the degree of charge ordering is considerably reduced from the ideal values.

Also, we have refined the SXRPD pattern obtained at $150 \mathrm{~K}$ on the basis of the bondcentred model. We have used as starting atomic coordinates those reported in the work of Daoud-Aladine et al. for the $\mathrm{Pr}_{0.6} \mathrm{Ca}_{0.4} \mathrm{MnO}_{3}$ perovskite [9].

The obtained lattice parameters, refined atomic coordinates, derived interatomic distances and agreement factors are summarized in Table 5 and Table 6.

Table 5. Structural parameters after the Rietveld refinement of the SXRPD pattern with the bond-centred model (S.G.: Pmn2 1 ) of the data obtained at $T=150 \mathrm{~K}$ The estimated errors are given in parentheses.

\begin{tabular}{|c|c|c|c|c|}
\hline \multirow[t]{2}{*}{ Atom } & \multicolumn{3}{|c|}{$a=7.6707(1) \AA, b=10.8521(2) \AA ̊ ., c=5.4405(1) \AA$} & \multirow[t]{2}{*}{$U /\left(\AA^{2}\right)$} \\
\hline & $x$ & $y$ & $z$ & \\
\hline $\mathrm{Pr} / \mathrm{Na}(1)$ & 0.0 & $0.8869(12)$ & $0.4901(22)$ & $0.0039(5)$ \\
\hline $\mathrm{Pr} / \mathrm{Na}(2)$ & 0.0 & $0.6366(12)$ & $0.0127(21)$ & $0.0039(5)$ \\
\hline $\mathrm{Pr} / \mathrm{Na}(3)$ & 0.0 & $0.1385(13)$ & $-0.0014(22)$ & $0.0039(5)$ \\
\hline $\mathrm{Pr} / \mathrm{Na}(4)$ & 0.0 & $0.3886(12)$ & $0.5057(22)$ & $0.0039(5)$ \\
\hline $\mathrm{Mn}(1)$ & $0.250(4)$ & $0.8735(26)$ & 0.0 & $0.0030(6)$ \\
\hline $\operatorname{Mn}(2)$ & $0.749(4)$ & $0.3771(24)$ & $0.0094(25)$ & $0.0030(6)$ \\
\hline O1 & $0.285(6)$ & $0.983(4)$ & $0.685(6)$ & $0.0059(9)$ \\
\hline $\mathrm{O} 2$ & $0.808(4)$ & $0.265(4)$ & $0.263(7)$ & $0.0059(9)$ \\
\hline O3 & $0.722(7)$ & $0.227(5)$ & $0.794(6)$ & $0.0059(9)$ \\
\hline $\mathrm{O} 4$ & $0.223(5)$ & $0.506(5)$ & $0.248(6)$ & $0.0059(9)$ \\
\hline O5 & 0.0 & $0.126(9)$ & $0.551(9)$ & $0.0059(9)$ \\
\hline O6 & 0.0 & $0.860(8)$ & $0.951(9)$ & $0.0059(9)$ \\
\hline $\mathrm{O} 7$ & 0.0 & $0.384(8)$ & $0.938(9)$ & $0.0059(9)$ \\
\hline \multirow[t]{2}{*}{$\mathrm{O} 8$} & 0.0 & $0.616(7)$ & $0.593(9)$ & $0.0059(9)$ \\
\hline & \multicolumn{4}{|c|}{$R_{\mathrm{wp}}=4.72, R_{\mathrm{p}}=3.91, X^{2}=2.93$} \\
\hline
\end{tabular}


Table 6. Selected interatomic distances $(\AA)$ in $\operatorname{Pr}_{0.79} \mathrm{Na}_{0.21} \mathrm{MnO}_{3}$ at $T=150 \mathrm{~K}$ obtained from the Rietveld refinement to the bond-centred model and valences determined from the Bond Valence model for $\mathrm{Mn}$ atoms The errors are given in parentheses.

\begin{tabular}{|l|l|}
\hline & Bond length $(\AA)$ \\
\hline Mn1-O1 & $2.098(21)$ \\
\hline Mn1-O1 & $1.878(24)$ \\
\hline Mn1-O2 & $2.028(24)$ \\
\hline Mn1-O3 & $1.952(20)$ \\
\hline Mn1-O5 & $1.94(4)$ \\
\hline Mn1-O6 & $1.94(4)$ \\
\hline BVS (Mn1) & 3.23 \\
\hline Mn2-O2 & $1.896(22)$ \\
\hline Mn2-O3 & $2.012(20)$ \\
\hline Mn2-O4 & $1.916(19)$ \\
\hline Mn2-O4 & $1.921(21)$ \\
\hline Mn2-O7 & $1.966(34)$ \\
\hline Mn2-O8 & $1.966(34)$ \\
\hline BVS (Mn2) & 3.44 \\
\hline
\end{tabular}

In this orthorhombic structure (S.G.: $P 2_{1} n m$ ), there are two non-equivalent crystallographic sites for the Mn cations in (4b) Wyckoff sites. We have calculated the charges at these two sites using the BVS method, finding that they are different: 3.23 for $\mathrm{Mn}(1)$ versus 3.44 for $\mathrm{Mn}(2)$ (see Table 6). This is an interesting result as the starting point for this refinement has been the bond centred model, in which-in principle-Mn cations are expected to present the same charge at the two sites. This result would then imply that in this compound the extra electron would not be symmetrically localized in the $\mathrm{Mn}-\mathrm{O}-\mathrm{Mn}$ bonds but asymmetrically distributed in the Mn-pairs.

And the resulting electronic picture is in fact not so different from that obtained when using the Radaelli's model. In that case, starting form a full site-centred model, the results indicate that the degree of localization is much smaller than expected even if there is a difference in charge between the two Mn sites.

The agreement factors do not allow to rule out any of these two refinements, they are very similar (seeTable 3 and Table 5 ) and there is not a significant difference between them, even if those based on the Radaelli's model are slightly better. On the basis of this result and the electronic pictures obtained when starting form these two extreme models, it seems very plausible that the real situation is intermediate between the two of them: neither purely site centred nor purely bond centred, but in-between as in the Efremov's model for near half-doped magnites [10]. 
In any case, to further clarify this point a detailed structural characterization on a single crystal and highly resolved neutron diffraction studies [23] would be most useful.

\subsection{Dielectric properties}

The relative complex permittivity of this sample, $\varepsilon_{\mathrm{T}}(\omega)=\varepsilon_{\mathrm{r}}^{\prime}(\omega)-\mathrm{i} \varepsilon_{\mathrm{T}}^{\prime \prime}(\omega)$, was measured as a function of frequency and temperature both with gold and with silver sputtered contacts. As the obtained results are similar in both cases, in what follows we will present the dielectric behaviour observed independently of the type of contact used, unless specifically indicated.

Fig. 3a shows the frequency dependence of the real part of the permittivity, the socalled dielectric constant, ${ }_{s}^{0}$, measured at different temperatures. As it can be seen, in the low frequency range $\left(v<10^{3} \mathrm{~Hz}\right)$ and for a given temperature, the dielectric constant markedly decreases with frequency especially for $T>150 \mathrm{~K}$. Meanwhile for $v>10^{3} \mathrm{~Hz}$, the frequency and temperature dependence of ${ }_{x}^{b}$ is much smaller.

Fig. 3.

Frequency dependence of the (a) dielectric constant and (b) imaginary part of the dielectric permittivity measured in the temperature range $90<T(\mathrm{~K})<300$ using sputtered gold contacts.

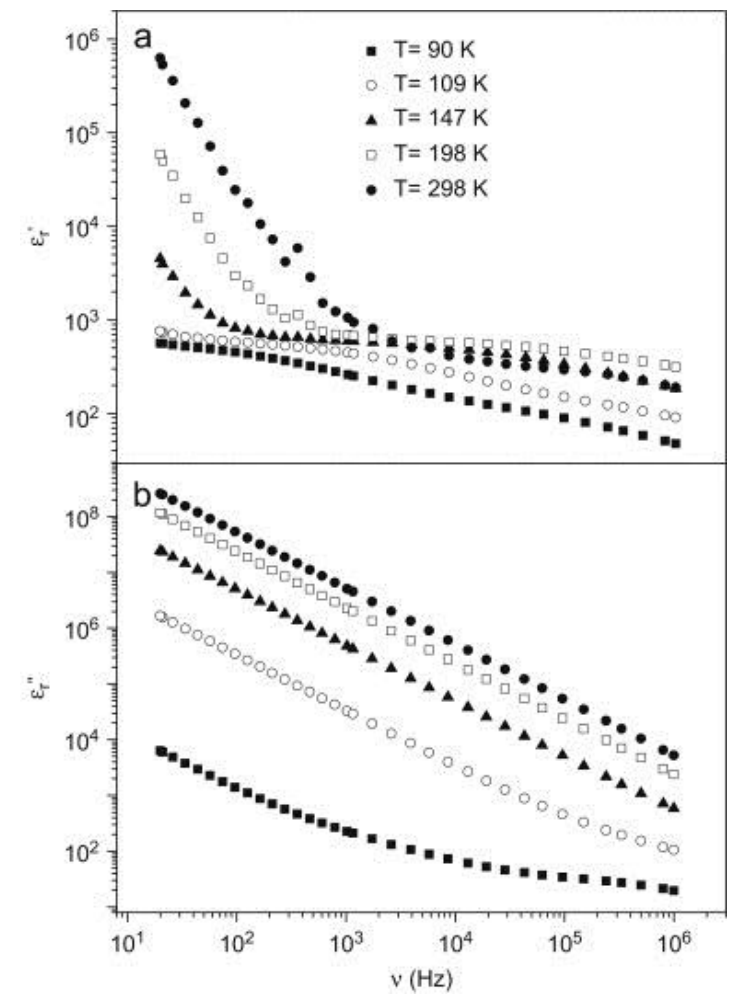

Meanwhile, the imaginary part of the dielectric permittivity, ${ }_{s}^{m}$, shows rather high values that decrease as frequency increases and as temperature decreases (see Fig. 3b).

On the other hand, Fig. 4a shows the temperature dependence of the dielectric constant, $u_{s}$, obtained under different measuring frequencies and using gold contacts. 
As it can be seen ${ }_{s}$, that shows small values at low temperatures, increases significantly as temperature gets higher. And most interesting, $\varepsilon_{\mathrm{T}}^{\prime}(T)$ shows a broad maximum around the charge ordering temperature $T_{\mathrm{CO}}=T_{\mathrm{M}} \sim 220 \mathrm{~K}$, to then decrease as temperature rises further.

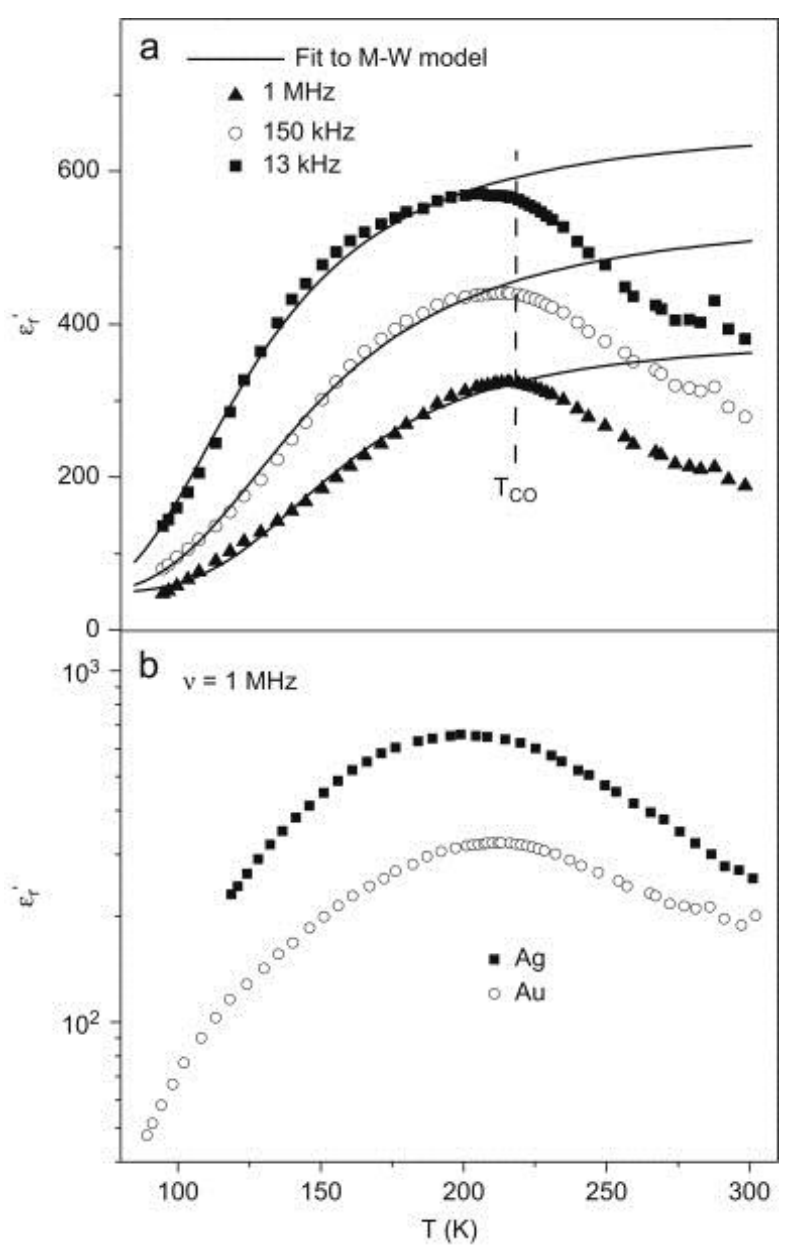

Fig. 4. (a) Temperature dependence of the dielectric constant measured at different frequencies with sputtered gold contacts, where the scatter symbols represent the experimental data and the solid lines correspond to fits to the Debye equation; and (b) $\varepsilon_{\mathrm{T}}^{\prime}(T)$ curves obtained using sputtered gold or sputtered silver contacts both revealing the presence of a maximum around $T_{\mathrm{CO}}$.

It should also be highlighted that although the value of the dielectric constant is seen to markedly vary with frequency, the position of the mentioned broad peak does not depend on frequency.

Such a behaviour would be intermediate between that of a conventional FE material (sharp peaks that are frequency independent) and a ferroeletric-relaxor (broad peaks and frequency dependent) [24].

Another very important remark is that the values of the dielectric constant are similar when using silver sputtered contacts, and that the anomaly of $\varepsilon_{\mathrm{r}}^{\prime}(T)$ persists even if the 
nature of the metal contacts changes, as shown in Fig. 4b. This result is a clear proof that the anomaly observed is not a surface effect, but intrinsic to the sample.

To deepen further into the dielectric behaviour of this compound we have performed impedance complex plane ( $Z^{\prime \prime}$ versus $Z$ ) analysis of the obtained data. This is a very powerful tool to separate the contribution from electrically heterogeneous materials [25] and [26].

Typical impedance complex plane plots corresponding to the temperature interval $90 \leqslant T(\mathrm{~K})<120$ and $120 \leqslant T(\mathrm{~K}) \leqslant 300$ are shown in Fig. $5 \mathrm{a}$ and $\mathrm{b}$, respectively.
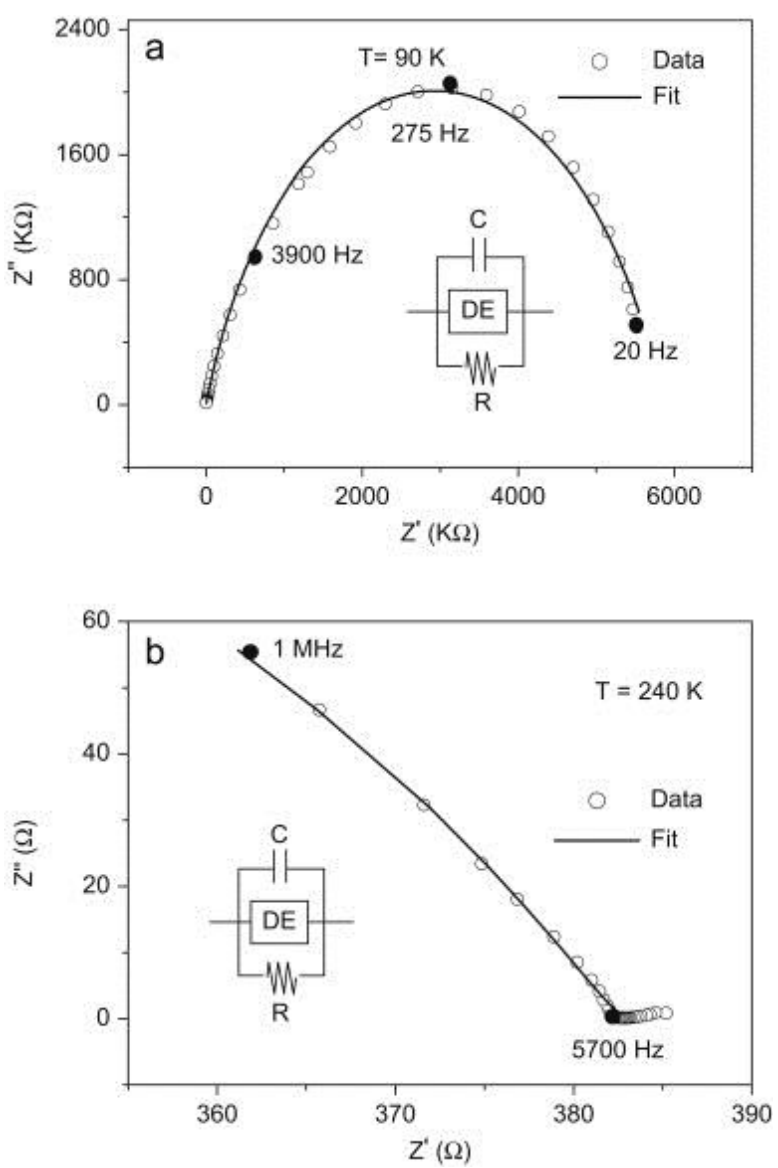

Fig. 5.

Typical impedance complex plane plots of $\mathrm{Pr}_{0.79} \mathrm{Na}_{0.21} \mathrm{MnO}_{3}$, corresponding fits and equivalent circuits: (a) for the lower temperature region $(90 \leqslant T(\mathrm{~K}) \leqslant 120)$ and $(\mathrm{b})$ for the higher temperature region $(120<T(K) \leqslant 300)$. The open circles represent the experimental data and the solid line the corresponding fits.

As it can be seen, in the range $90 \leqslant T(K)<120$ they show a single large arc, while for $T \geqslant 120 \mathrm{~K}$ a second small arc appears additionally in the low frequency range.

The large arc observed in the temperature range $90 \leqslant T(K)<120$ can be modelled by an equivalent circuit containing three elements connected in parallel: a resistance $(R)$ and a capacitance $(C)$, that is frequency independent, and a frequency dependent distributive element (DE). This DE element is modelled as an impedance inversely 
proportional to a power of the frequency [21] and the fitted frequency exponent of this $D E$, that depends slightly on temperature, is around 0.8. As this large arc goes to zero and the order of magnitude of its $C$ is of $\mathrm{pF} \mathrm{cm}^{-1}$, it means that in this temperature interval and in the frequency range studied only the intrinsic response is being observed.

On the other hand, the plots obtained at higher temperatures $(T \geqslant 120 \mathrm{~K})$, show two arcs ( Fig. 5b): a large arc that can be modelled by the equivalent circuit described before; and an additional small arc at low frequencies, that it is too small for fits. As this second small arc does not intercept the origin coordinates, it should have an extrinsic origin like grain boundaries or electrode effects.

Therefore, in this higher temperature range $120 \leqslant T(K) \leqslant 300$, the dielectric constant contains both an intrinsic and an extrinsic contribution. The former, due to the bulk material governs the response observed at medium and high frequencies, while extrinsic factors mainly contribute at low frequencies.

Taking into account these results, we can try to explain the obtained data on the basis of the Maxwell-Wagner (M-W) model, which refers to interfacial polarization occurring in electrically inhomogeneous systems [27]. In this context, it is known that when an electric current passes through interfaces between two different dielectric media, because of their different conductivities surface charges pile up at the interfaces [28]. And, the system shows a Debye-like relaxation process under an external alternating field, even if without any dipole relaxation, the so-called M-W relaxation [27].

Such Debye-like relaxation can be expressed as [27]:

$$
\mathscr{L}_{1}^{\prime}=\varepsilon_{\infty 0}+\frac{\Sigma_{\alpha}-\varepsilon_{\infty 0}}{1+\omega^{2} \tau^{2}}
$$

where $\varepsilon_{\infty}$ is the value of dielectric constant at high frequency, $\varepsilon_{\mathrm{s}}$ the value of dielectric constant at low frequency, $\omega$ the angular frequency and $t$ is the relaxation time.

Talking into account that the thermal dependence of $T$ is of the Arrhenius type:

$$
\tau=\tau_{0} \mathrm{e}^{A / k T}
$$

where $T_{0}$ is a constant, $\Delta$ the activation energy and $k$ is the Boltzmann's constant. 
We have tried to fit our $\varepsilon_{\mathrm{r}}^{\prime}(T)$ data to Eq. (1). As shown in Fig. 4, the calculated data can fit only partially the experimental ones: they allow to explain the observed increase in from low temperature, but not the presence of the maximum around $T$ co. Therefore, the maximum in $\varepsilon_{\mathrm{T}}^{\prime}(T)$ cannot be attributed to the presence of extrinsic factors, and must have its origin in the material's intrinsic response.

The impedance spectroscopy results corroborate that the maximum observed in the ${ }_{x}$ versus temperature curve has an intrinsic origin: this peak is also observed when measuring using high frequencies, and as said before in such conditions the observed dielectric behaviour is only due to the material's bulk response as the extrinsic factors no longer contribute.

Now, the question is why the dielectric constant shows an anomaly at $T$ co. We suggest that it is related to the charge condensation that is taking place at that temperature that could be giving rise to the formation of polar entities in the material. From the fact that the peak of $x_{T}$ around $T_{\mathrm{CO}}$ is very broad, similar to the behaviour displayed by relaxors, we deduce that such phenomenon could be local in the form of small polar clusters or domains.

The origin of such polar zones could be an asymmetric charge distribution as proposed by Efremov et al. for near half-doped manganites [10]: a charge ordering that is intermediate between site-centred and bond-centred, as the structural characterization by SXRPD seems to suggest.

Summarizing, we have prepared the $\operatorname{Pr}_{0.79} \mathrm{Na}_{0.21} \mathrm{MnO}_{3}$ compound by a solid-state process. We have carried out its structural characterization by SXRPD at room temperature and at $150 \mathrm{~K}\left(T<T_{\mathrm{CO}}\right)$. In the CO state, the SXRPD data can be refined both on the basis of the site-centred model and the bond-centred model proposed for half-doped manganites, without a clear advantage of one model over the other. Evermore, it is very plausible that the real situation is intermediate between these two extremes, as the obtained results seem to indicate.

From the dielectric point of view, at the CO temperature shows a maximum in the dielectric constant whose origin is intrinsic, and cannot be attributed to the presence of extrinsic factors.

We relate this dielectric behaviour to the formation of polar entities at the temperature of charge condensation, due to an asymmetric charge distribution-intermediate between site-centred and bond-centred-of the type described by Efremov et al. for near half-doped manganites [10]. 


\section{Acknowledgments}

The authors are grateful for financial support from MEC (Spain) under Project FEDER MAT 2004-05130 and from Xunta de Galicia under Project PGIDIT06PXB103298PR. S. Yáñez-Vilar wants to thank to MEC of Spain for her FPI fellowship and M. SánchezAndújar acknowledges Xunta de Galicia for support under program Parga Pondal. We also thank the European Synchrotron Radiation Facility for provision of synchrotron radiation facilities and Germán Castro for his assistance in using beamline BM25A.

\section{References}

1. C.N.R. Rao, B. Raveau

Charge Ordering and Related Properties of Manganese Oxides

World Scientific, Singapore (1998)

2. E. Dagotto, T. Hotta, A. Moreo

Phys. Rep., 344 (2001), pp. 1-153

3. G. Subías, J. García, M.G. Proietti, J. Blasco

Phys. Rev. B, 56 (1997), pp. 8183-8191

4. F. Rivadulla, E. Winkler, J.-S. Zhou, J.B. Goodenough

Phys. Rev. B, 66 (2002), pp. 174432-174442

5. J.B. Goodenough

Phys. Rev., 100 (1954), pp. 564-573

6. P.G. Radaelli, D.E. Cox, M. Marezio, S.-W. Cheong

Phys. Rev. B, 55 (1997), pp. 3015-3023

7. P.M. Woodward, D.E. Cox, T. Vogt, C.N.R. Rao, A.K. Cheetham

Chem. Mater., 11 (1999), pp. 3528-3538

8. J.P. Attfield

Solid State Sci., 8 (2006), pp. 861-867

9. A. Daoud-Aladine, J. Rodriguez-Carvajal, L. Pinsard-Gaudart, M.T. Fernandez-Diaz, A. Revcolevschi

Phys. Rev. Lett., 89 (2002), pp. 97205-99729

10. D.V. Efremov, D.I. Khosmkii, J. Brink

Nat. Mat., 3 (2004), pp. 853-856

11. C. Jardón, F. Rivadulla, L.E. Hueso, A. Fondado, J. Rivas, M.A. López Quintela, R. Zysler, M.T. Causa, P. Sande

J. Magn. Magn. Mater., 196/197 (1999), pp. 475-476

12. S. Mercone, A. Wahl, A. Pautrat, M. Pollet, C. Simon 
Phys. Rev. B, 69 (2004), pp. 174433-174439

13. J. Rivas, B. Rivas-Murias, A. Fondado, J. Mira, M.A. Señarís-Rodríguez Appl. Phys. Lett., 85 (2004), pp. 6224-6226

14. J. Hejtmánek, Z. Jirák, J. Sebek, A. Strejc, M. Hervieu J. Appl. Phys., 89 (2001), pp. 7413-7415

15. T. Satoh, Y. Kikuchi, K. Miyano, E. Pollert, J. Hejtmánek, Z. Jirák Phys. Rev. B, 65 (2002), pp. 125103-125107

16. Z. Jirák, J. Hejtmánek, K. Knízek, M. Marysko, E. Pollert, M. Dlouhá, S. Vratislav, R. Kuzel, M. Hervieu

J. Magn. Magn. Mater., 250 (2002), pp. 275-287

17. A.C. Larson, R.B. von Dreele, General Structure Analysis System (GSAS), Los Alamos National Laboratories, Report LAUR 86-748, 1990.

18. I.D. Brown, D. Altermatt

Acta Crystallogr. B, 41 (1985), pp. 240-247

19. St. Adams

Acta Crystallogr. B, 57 (2001), pp. 278-287

20. R. Viana, P. Lunkenheiner, J. Hemberger, R. Böhmer, A. Loidl

Phys. Rev. B, 50 (1994), pp. 601-604

21. J. Ross Macdonald, LEVM Version 8.0 Complex Nonlinear Squares Fitting Program, 2003.

22. D.P. Kozlenko, Z. Jirak, I.N. Goncharenko, B.N. Savenko

J. Phys.: Condens. Matter, 16 (2004), pp. 5883-5895

23. R.J. Goff, J.P. Attfield

Phys. Rev. B, 70 (2004), pp. 140404-140408

24. G.A. Samara

J. Phys.: Condens. Matter, 15 (2003), pp. R367-R411

25. E. Barsoukov, J. Ross Macdonald

Impedance Spectroscopy: Theory, Experiment and Applications

Wiley, NJ (2005)

26. J. Ross Macdonald Impedance Spectroscopy: Emphasizing Solid Materials and Systems

Wiley (1987)

27. A. Von Hippel Dielectrics and Waves

Artech House, Boston (1995)

28. E. Billing, K.W. Plessner

Proc. Phys. Soc. Lond., 64B (1951), pp. 361-363 